\title{
A Study of Incidence and Significance of Intraoperative Peritoneal fluid Fungal Culture in Patients with Perforated Peptic Ulcers
}

\author{
Mahim Koshariya, Surabhi Garg, Anshul Siroliya, Abhishek Shitole, Rakesh Pandey, Amit Katlana, \\ Arvind Rai
}

Corresponding author:

Dr.Mahim Koshariya

MS,FMAS,FIASGO,FIAGES,FAIS,FALS

Professor

Department of Surgery

Gandhi Medical College and Hamidia

Hospital Bhopal, 462001, India

Tel. +919826031583

E mail : mahimk2000@yahoo.co.uk

\section{Abbreviations:}

APACHE - Acute Physiology And Chronic Health Evaluation; MPI - Mannheim Peritonitis Index; PPU - Perforated Peptic Ulcer.
Department of Surgery, Gandhi Medical College and Hamidia Hospital Bhopal, India

\section{ABSTRACT}

Background: Although the incidence of peptic ulcer disease has reduced, the peptic ulcer perforation rates remain constant. Till recently the emphasis has been placed on the identification of microbial flora associated with peritonitis caused by perforated peptic ulcer. The aim of this study was to determine the incidence and significance of intraoperative peritoneal fluid culture of fungus in patients with perforated peptic ulcers.

Materials and method: In this study, we included 53 patients with intraoperatively confirmed perforated gastroduodenal ulcers admitted in our hospital. Patients were evaluated pre-operatively; intra-operative peritoneal fluid specimen was sent for culture \& sensitivity; post-operative records of various parameters were studied; patients' morbidity and mortality were evaluated with reference to their culture outcome.

Results: Fungal cultures of peritoneal fluid were positive in 24 out of 53 patients (45.2\%), Candida being the most common isolated species in 22 patients (91.6\%), followed by Apergillus. Fungal cultures were found positive more commonly in patients above 50 years of age and in females. Age, preoperative organ failure, delay in operation, high Mannheim Peritonitis Index (MPI) and Acute Physiology And Chronic Health Evaluation (APACHE) II scores, smoking, alcohol abuse, steroid use, $\mathrm{H} 2$ blockers and preoperative antibiotic therapy were risk factors for a positive fungal culture. MPI of 20 or more was statistically significant $(p<0.001)$. Increased morbidity was observed in fungal peritonitis patients in comparison to non-fungal peritonitis cases. The Candida peritonitis group had a mortality of $18.18 \%$, while the mortality in the nonCandida peritonitis group was $3.44 \%$.

Conclusion: Fungal positivity was a significant risk factor for adverse outcome in patients with a PPU. Patients with associated risk factors and a MPI score $>24$ and APACHE II score of $>12$ with positive intra-operative peritoneal fluid fungal culture can be considered for early antifungal treatment.

Key words: perforated peptic ulcer (PPU), positive peritoneal fungal culture, candida peritonitis, antifungal therapy

\section{INTRODUCTION}

The gastrointestinal tract is a major reservoir of microorganisms and an important portal for intraabdominal infections and sepsis (1). Studies have 
shown that composition of the microbial flora in the abdominal fluid varies depending on the location of the perforation. Ruiter et al reported the maximum proportion of Candida-positive cultures in patients with peptic perforation (41\%) (2). Perforated peptic ulcer (PPU) is a surgical emergency and is associated with short-term mortality and morbidity in up to 30 and $50 \%$ of patients, respectively (3). Until recently, the leading pathogens associated with secondary peritonitis were Gram-negative and anaerobic bacteria. Fungal infection has become more common in recent years especially in critically ill patients in intensive care (4). Also, positive peritoneal fungal culture has been shown to have increased morbidity and poor prognosis in patients with peptic ulcer perforation. Despite the association between high mortality rates and positive peritoneal fungal cultures in patients with perforated peptic ulcer, conflicting views about the significance of fungal infection still exist (5). Overall, fungal peritonitis accounts for approximately $3 \%-12 \%$ of all cases of peritonitis in adults, but the rate varies largely between different centers (6). The most common cause of fungal peritonitis is Candida. Without effective treatment, Candida peritonitis can lead to systemic infection, multiorgan failure, and death. The mortality of Candida peritonitis is very high, ranging from $20 \%$ - $70 \%$ (7). This study was undertaken in order to study the incidence of fungalpositive peritoneal cultures in patients with perforated peptic ulcers and determine its significance.

\section{MATERIALS AND METHOD}

The study was designed as a prospective study of 53 cases of perforated gastric \& duodenal ulcers, admitted in the Department of Surgery, Gandhi Medical College and associated Hamidia Hospital Bhopal in 2004 and 2005. Intra-operative peritoneal fluid fungal cultures were taken in all cases and their significance in relation to post-operative morbidity and mortality was analysed. A written consent was taken from every patient prior to management.

All traumatic blunt or penetrating hollow viscous injuries, terminal small bowel and colonic perforations, patients aged < 16 yrs of age, as well as those dying within $48 \mathrm{hrs}$ of surgery were excluded. All patients included in study were followed from the time of admission, to their clinical progress \& management employed, till the final outcome. Subsequently the patients were followed for 1 month after discharge.

Pre-operative clinical data was recorded, including age, sex, condition on admission, duration of signs and symptoms, pre-operative use of antibiotics and duration of operation. For each patient a MPI score \& APACHE II score was assigned. From an operative standpoint, most of the patients were managed by simple closure (Graham's patch) and drainage, while definitive surgery was performed in only two patients.

An intraabdominal specimen was obtained for fungal culture at the time of laparotomy. Subsequent cultures from both the drain and the main wound were sent if the first culture came out to be positive. Blood for fungal culture was taken only when the patient's temperature was above $39 \circ \mathrm{C}$. All the specimens were cultured on Sabouraud Dextrose Agar as a selective medium and incubation was done at $37^{\circ} \mathrm{C}$ for 48 hours. Lactophenol Cotton Blue mount showing budding yeast cells from the colonies obtained on SDA were identified by conventional methods. Growth of organisms was recorded as mild, moderate or heavy.

Postoperative daily records of pulse, temperature, $\mathrm{BP}$, respiration, output, etc were noted. The outcomes were determined from case notes, which included duration of stay, duration of central line, morbidity, surgical site infection and mortality.

\section{RESULTS}

The patient population comprised 53 cases of perforated peptic ulcers (confirmed intra-operatively), out of which $66 \%$ were gastric and $34 \%$ were pyloroduodenal. Females showed a slightly higher incidence of perforated gastric ulcers than males, while the inverse situation was true in cases of perforated duodenal ulcers. However, overall peptic ulcer perforations were more common in males as compared to females, with an average male: female ratio of 6.5:1

The incidence of fungal peritonitis in the study group was quite significant (45.2\%), with Candida being the most frequently isolated species (41.51\%). Fungal culture positivity in relation to gastric perforation was $40 \%$, while in relation to perforated duodenal ulcers it was $55.5 \%$. These values suggested a higher incidence of fungal peritonitis in cases of perforated duodenal ulcers (table 1).

Patients older than 50yrs were found to have a higher risk for positive fungal culture, whereas patients younger than $30 y$ rs were least affected.

History of PUD, steroid intake, alcohol consumption, use of $\mathrm{H} 2$-blockers and smoking were associated with an increased risk of fungal peritonitis. Also, there was an increased risk of fungal peritonitis in cases of delayed presentations (4.2 days in average), longer duration of pre-operative antibiotics (2.41 days in average) and in patients with organ failure (66.67\%) (table 2). 
Table 1 - Incidence of positive fungal cultures

\begin{tabular}{|c|c|c|c|c|c|c|c|}
\hline \multicolumn{2}{|c|}{ S.No. } & \multicolumn{2}{|c|}{$\begin{array}{c}\text { Total Cases n=53 } \\
\text { No. Percentage }\end{array}$} & \multicolumn{2}{|c|}{$\begin{array}{c}\text { Gastric Ulcers } \\
n=35\end{array}$} & \multicolumn{2}{|c|}{$\begin{array}{c}\text { Duodenal Ulcers } \\
n=18\end{array}$} \\
\hline 1 & Positive Fungal Cultures & 24 & $45.2 \%$ & 14 & $40 \%$ & 10 & $55.55 \%$ \\
\hline 2 & Positive Candida Cultures & 22 & $41.51 \%$ & 13 & $37.14 \%$ & 9 & $50 \%$ \\
\hline 3 & Positive Aspergillus Cultures & 2 & $03.7 \%$ & 1 & $2.86 \%$ & 1 & $5.55 \%$ \\
\hline 4 & None & 29 & $54.8 \%$ & & & & \\
\hline
\end{tabular}

Univariate analysis revealed that an MPI score $>26$ and an APACHE II score $>12$ were significantly associated with positive fungal cultures. Moreover, patients with systemic diseases had raised incidence of Candida peritonitis. Prolonged hospital stay (19.93 days in average), as well as a longer duration of central line (12.12 days in average) were found in patients positive for fungal culture in comparison to fungal negative patients. Local wound infection resulting in increased morbidity was found to be significantly higher (Chi Square test $-p<.05)$ in the fungal positive group $(100 \%)$ as compared patients with a negative culture (20.68\%).

It was also noted that patients with fungal peritonitis have a higher mortality rate $(45.83 \%)$ than those with non-fungal peritonitis $(3.44 \%)$ (Chi Square test $p<.05)$ (table 3).

\section{DISCUSSION}

Peptic ulcer perforation is a commonly encountered surgical emergency in India. The gastrointestinal tract is a major reservoir for organisms and a source of intra- abdominal infections and sepsis. Ruiter et al. reported the maximum rate of isolation of Candida occurred in cases of gastric perforation (41\%), followed by small gut perforation (34.1\%) (2). The main goal of the present study was to obtain an overview of the frequency and significance of the recovery of positive fungal cultures from intra-abdominal specimens after peptic perforation.

In our series the total incidence of peptic perforationrelated fungal peritonitis came out to be $45.2 \%$, i.e. 24 out of 53 cases. Amongst these, 22 cultures showed Candida $(41.51 \%)$ as an isolated species, while the other two were positive for Aspergillus species (3.7\%) as well.

The youngest patient was of 16 years old, while the eldest one was 70 years of age. The highest incidence of perforated peptic ulcers was found in the 30-49 years age group (39.6\%), followed by the age group of $\geq 50 y r s$ $(37.7 \%)$. The mean age when peptic ulcer perforation occurred in the present series was 43.32 years. However, the incidence of positive peritoneal fluid fungal culture was found to be highest in $\geq 50$ years age group ( $45.83 \%)$, followed by the $30-49$ years age group

Table 2 - Incidence of risk factors related to positive fungal culture

\begin{tabular}{|c|c|c|c|c|c|c|c|c|c|c|c|c|c|c|c|}
\hline \multirow[t]{2}{*}{ S.No } & & \multicolumn{2}{|c|}{$\begin{array}{c}\text { HPUD } \\
n=15\end{array}$} & \multicolumn{2}{|r|}{$\begin{array}{c}\text { Steroid } \\
n=9\end{array}$} & \multicolumn{2}{|c|}{$\begin{array}{c}\text { Smoking } \\
n=27\end{array}$} & \multicolumn{2}{|c|}{$\begin{array}{c}\text { Alcohol } \\
n=17\end{array}$} & \multicolumn{2}{|c|}{$\begin{array}{c}\text { H2 Blocker } \\
n=12\end{array}$} & \multirow[t]{2}{*}{$\begin{array}{c}\text { DOS } \\
\text { Avg-3days }\end{array}$} & \multirow[t]{2}{*}{$\begin{array}{c}\text { Pre-op } \\
\text { Antibiotics }\end{array}$} & \multicolumn{2}{|c|}{$\begin{array}{l}\text { Organ } \\
\text { Failure }\end{array}$} \\
\hline & & & & & & & & & & & & & & $\mathrm{n}=18$ & $\%$ \\
\hline 1 & $\begin{array}{l}\text { Positive } \\
\text { Fungal }\end{array}$ & & & & & & & & & & & & & & \\
\hline & Cultures & 9 & $60 \%$ & 5 & $55.55 \%$ & 10 & $37.03 \%$ & 9 & $52.94 \%$ & 8 & $75 \%$ & 4.2 days & 2.41 & 12 & $66.67 \%$ \\
\hline 4 & None & 6 & $40 \%$ & 4 & $44.45 \%$ & 17 & $62.97 \%$ & 8 & $47.06 \%$ & 4 & $25 \%$ & 2 days & 0.86 & 6 & $33.33 \%$ \\
\hline
\end{tabular}

Table 3 - Severity score, systemic disease \& their relation with fungal outcome

\begin{tabular}{|c|c|c|c|c|c|c|c|c|c|}
\hline \multicolumn{2}{|c|}{$\begin{array}{l}\text { S. } \\
\text { No. }\end{array}$} & \multirow{2}{*}{$\begin{array}{c}\begin{array}{c}\text { MPI } \\
\text { Score }\end{array} \\
22.26 \\
\end{array}$} & \multirow{2}{*}{$\begin{array}{c}\text { APACHE II } \\
\text { Score } \\
13\end{array}$} & \multicolumn{2}{|c|}{$\begin{array}{c}\text { Systemic Disease } \\
n=53\end{array}$} & \multirow{2}{*}{$\begin{array}{c}\begin{array}{l}\text { Duration of } \\
\text { Stay (Days) }\end{array} \\
14.5\end{array}$} & \multirow{2}{*}{$\begin{array}{c}\begin{array}{c}\text { CVP Line } \\
\text { (Days) }\end{array} \\
7.68\end{array}$} & \multirow{2}{*}{$\begin{array}{c}\begin{array}{c}\text { Wound Infection } \\
\mathrm{n}=\mathbf{5 3}\end{array} \\
30\end{array}$} & \multirow{2}{*}{$\begin{array}{c}\begin{array}{c}\text { Mortality } \\
\mathbf{n}=\mathbf{5 3}\end{array} \\
12\end{array}$} \\
\hline 1 & Total Avg/Number & & & 18 & $33.96 \%$ & & & & \\
\hline 2 & Positive Fungal Culture & 28.08 & 15 & 13 & $72.23 \%$ & 19.93 & 12.12 & 24 & 11 \\
\hline 3 & None & 17.44 & 9 & 5 & $27.77 \%$ & 11.96 & 4 & 6 & 1 \\
\hline
\end{tabular}


(37.5\%) and least in the $10-29$ years age group, i.e. $16.67 \%$. Age $>60$ years seems to be an independent risk factor for fungal peritonitis and is significantly related to a positive intra-peritoneal fungal culture (5). The incidence of fungal peritonitis was found to be higher in female patients (71\%) in comparison to males (41\%). However, the number of cases in the former group was quite small, which renders this difference not statistically significant. Therefore, a larger trial is recommended in order to achieve statistical significance.

As far as the predisposing factors were concerned in the present series, about $50 \%$ of the patients were smokers, out of which $37 \%$ had positive a fungal culture. 9 / 24 fungal positive cases in our series were alcoholic. It has conclusively been shown that smoking delays healing and promotes recurrent peptic ulcers, which increases risk of fungal colonization $(8,9)$. Both smoking and alcohol abuse are associated with compromised host defenses and hence with high incidence of fungal culture. In our series, 9 patients received steroid preoperatively, out of which 5 had fungal peritonitis (55.55\%). Other studies have also shown that steroids and pre-operative $\mathrm{H}-2$ receptor therapy impair healing of peptic ulcer and promote fungal colonization of the stomach (8).

The combination of prolonged duration of symptoms, preoperative antibiotics and preoperative organ failure creates an immune-compromised host with altered endogenous flora, which increases susceptibility for fungal infection of the peritoneal cavity in the event of peptic ulcer perforation $(5,9)$. The presence of systemic diseases and malignancies also results in an immunocompromised host with an increased susceptibility to opportunistic infections, especially fungal ones. In this series, $70 \%$ of the patients who were positive for fungal culture had some form of systemic disease; in comparison, only $30 \%$ patients with negative fungal culture had systemic disease. Patients with an MPI score of 26 or more and an APACHE II score of more than 12 were found to be more susceptible to fungal peritonitis. A high APACHE II (>13) and MPI score $>20$ have been shown as independent risk factors for Candida infection (5,11, $12,13)$. This subgroup of patients can be considered for early antifungal therapy.

The mean length of stay in the hospital for patients having fungal peritonitis was 20 days, in comparison to 12 days in the nonfungal peritonitis group. The mean duration for in situ CVP line in our study was 7.7 days. The patients with fungal peritonitis had a mean of 12 days in comparison to the mean of 4 days in non-fungal peritonitis patients. This is an indisputable indicator of serious postoperative problems and shows a protracted postoperative course with an increase in the expenditures (5). Local wound infection resulting in increased morbidity was found to be significantly higher in the positive fungal culture group (100\%). In contrast, only $20.68 \%$ in the negative fungal culture group had wound infection.

In our study, a total of 12 patients died, rendering an overall mortality of $22.64 \%$. 11 deaths were seen in fungal peritonitis cases, while only 1 patient died due to non-fungal peritonitis. Mortality in patients with fungal peritonitis $(45.83 \%)$ was comparatively higher than in non-fungal peritonitis cases (3.44\%). Mortality was also found to be higher in patients $\geq 50$ yrs.

\section{CONCLUSION}

Although the number of patients in the present study is small, several conclusions could be drawn. The incidence of positive peritoneal fluid fungal culture was $45.2 \%$, with Candida being the most commonly isolated species (91.66\% of the total positive cultures). Patients $\geq 50$ yrs showed the maximum percentage of fungal positive cultures (45.83\%), suggesting higher age to be a risk factor. Smoking, alcohol abuse, steroids, previous history of PUD, $\mathrm{H} 2$ blockers, preoperative organ failure, associated systemic disease and preoperative antibiotics were all found to be risk factors related to positive fungal cultures. Increased morbidity, in terms of prolonged hospital stay, wound infection, fever and mechanical ventilation, were observed in fungal peritonitis patients in comparison to non-fungal peritonitis cases. A mortality of $45.83 \%$ was observed in the fungal peritonitis group. In the non-Candida peritonitis group, however, mortality dropped to $3.44 \%$, with $75 \%$ of deaths occurring in patients older than $50 \mathrm{yrs}$. In conclusion, it can be suggested that patients with associated risk factors, an MPI score $>24$ and an APACHE II score of $>12$, as well as positive intra-operative peritoneal fluid fungal culture, can be considered for early antifungal treatment. Early recognition and treatment of fungal co-infection can potentially minimize the high mortality seen in these patients.

\section{REFERENCES}

1. Wacha H, Hau T, Dittmer R, Ohmann C. Risk factors associated with intraabdominal infections: a prospective multicenter study. Peritonitis Study Group. Langenbecks Arch Surg. 1999;384(1):2432

2. de Ruiter J, Weel J, Manusama E, Kingma WP, van der Voort PH. The epidemiology of intra-abdominal flora in critically ill patients with secondary and tertiary abdominal sepsis. Infection. 2009;37(6):522-27.

3. Møller MH, Adamsen S, Thomsen RW, et al. Multicentre trial of a perioperative protocol to reduce mortality in patients with peptic ulcer perforation. Br J Surg. 2011; 98(6):802-10. 
4. Calandra T, Bille J, Schneider R, Mosimann F, Francioli P. Clinical significance of Candida isolated from peritoneum in surgical patients. Lancet. 1989;2(8677):1437-40.

5. Shan YS, Hsu HP, Hsieh YH, Sy ED, Lee JC, Lin PW. Significance of intraoperative peritoneal culture of fungus in perforated peptic ulcer. Br J Surg. 2003;90(10):1215-9.

6. Matuszkiewicz-Rowinska J (2009) Update on fungal peritonitis and its treatment. Perit Dial Int 29 Suppl 2: S161-165.

7. Carneiro HA, Mavrakis A, Mylonakis E. Candida peritonitis: an update on the latest research and treatments. World J Surg. 2011; 35(12):2650-9. doi: 10.1007/s00268-011-1305-2.

8. Wolińska-Wcisło M, Budak A, Bogdał J, Trojanowska D, Stachura J. Effect of fungal colonization of gastric mucosa on the course of gastric ulcers healing. Medical science monitor: international medical journal of experimental and clinical research. 2001;7.
266-75.

9. Bayer AS, Blumenkrantz MJ, Montgomerie JZ, Galpin JE, Coburn JW, Guze LB. Candida peritonitis: Report of 22 cases and review of the English literature. Am J Med. 1976; 61(6):832-40.

10. Alden SM, Frank E, Flancbaum L. Abdominal candidiasis in surgical patients. Am Surg. 1989;55(1):45-9.

11. Grunau G, Heemken R, Hau T. Predictors of outcome in patients with postoperative intra-abdominal infection. Eur J Surg. 1996;162(8): 619-25.

12. Pittet D, Monod M, Suter PM, Frenk E, Auckenthaler R. Candida colonization and subsequent infections in critically ill surgical patients. Ann Surg. 1994;220(6):751-8.

13. Billing A, Frohlich D, Schildberg FW. Prediction of outcome using the Mannheim peritonitis index in 2003 patients. Peritonitis Study Group. Br J Surg. 1994;81(2):209-13. 